Die Ärztegesellschaft des Kantons Bern initiierte im Sommer 2005 das Projekt «Monitoring Notfalldienst Bern». Der nachfolgende Bericht zeigt die Resultate der Ende 2006 abgeschlossenen Studie. Die Abteilung DDQ der FMH unterstützte diese Arbeit methodisch.

Die FMH würdigt die geleistete Arbeit und weist darauf hin, dass der Bericht vor dem Hintergrund der damaligen Situation zu betrachten ist. Bei Beginn der Studie wurden die Probleme des ärztlichen Notfalldienstes zunehmend wahrgenommen und die Forderung nach Lösungsansätzen laut. In der Zwischenzeit wurden verschiedene Arbeiten zum ärztlichen Notfalldienst - so z.B. von der gemeinsamen Arbeitsgruppe von GDK und BAG - in Angriff genommen und erste Massnahmen bereits umgesetzt.

Die FMH begrüsst diese Entwicklung und ist der Meinung, dass angesichts der noch immer vorhandenen Probleme des ärztlichen Notfalldienstes weitere Massnahmen nötig sind.

Der Zentralvorstand der FMH

\title{
Der ärztliche ambulante Notfalldienst im Kanton Bern 2006
}

\section{Beat Gafnera, \\ Heinz Zimmermann ${ }^{b}$, \\ Martina Hersperger}

a Dr. med., Facharzt für Allgemeinmedizin FMH, Mitglied des Vorstandes und Projektleiter Notfalldienst der Ärztegesellschaft des Kantons Bern

b Prof. Dr. med., Chefarzt Notfallzentrum Inselspital, Wissenschaftlicher Sekretär der Ärztegesellschaft des Kantons Bern

c lic. phil., Leiterin Abteilung Daten, Demographie und Qualität der Verbindung der Schweizer Ärztinnen und Ärzte FMH

* Die vollständige Studie kann über die E-Mail-Adresse praxigaf@hin.ch bezogen werden.

Korrespondenz:

Dr. med. Beat Gafner

Zur Station 7

CH-3145 Niederscherli

praxigaf@hin.ch

\begin{abstract}
Einleitung
Der ärztliche ambulante Notfalldienst durchlebt eine Phase des Umbruches sowohl in der Einstellung der praktizierenden Ärzteschaft zum bisher als selbstverständlich angesehenen Notfalldienst als auch in der Inanspruchnahme von notfallmässig zu erbringenden Leistungen durch die Bevölkerung. Mit einer von der Ärztegesellschaft des Kantons Bern unter Mitarbeit der FMH durchgeführten Studie* über den ärztlichen Notfalldienst sollte Transparenz in der Organisation und dem Ausmass der Belastung geschaffen werden: Niedrige Raten an Patientenkontakten, hohe Präsenzzeiten und unzeitgemässe räumliche Gliederung der Notfalldienstkreise charakterisieren die aktuelle Situation.
\end{abstract}

\section{Methode}

Im März und April 2006 wurden im Kanton Bern in allen Notfalldienstkreisen täglich die Anzahl Konsultationen in der Arztpraxis, Telefonanrufe und Hausbesuche im Rahmen des Notfalldienstes gesammelt und ausgewertet. Zusätzlich wurden Daten zu Geschlecht, Alter, Facharzttitel, Zugehörigkeit zu Bezirksverein und Notfalldienstkreis, Anzahl Notfalldiensttage pro Jahr, Dauer der Notfalldienstperioden und Wochentag der Dienstleistung erfasst und mit der Wohnbevölkerung verglichen. In der Originalarbeit werden detaillierte Zahlen aus allen Notfalldienstkreisen und getrennt nach Konsultationen, Hausbesuchen und Telefonen dargestellt und nach Möglichkeit in Zusammenhang mit den erhobenen Demographiedaten gebracht.

\section{Service de garde ambulatoire dans le canton de Berne en 2006}

Le service de garde ambulatoire traverse une phase de remise en question. Considéré jusqu'à présent comme allant de soi, il est désormais envisagé différemment, aussi bien par les médecins dans leur pratique quotidienne que par la population dans sa demande de prestations d'urgence. L'étude menée par la Société de médecine du canton de Berne avec le soutien de la FMH visait à créer de la transparence dans l'organisation et à mesurer I'ampleur de la tâche. Elle montre que la situation actuelle est caractérisée par un faible pourcentage de contacts avec les patients, des heures de présence élevées et une répartition spatiale inadéquate des districts de service de garde.

\section{Resultate}

\section{Rücklauf, Charakteristika} der Notfalldienstkreise

Bei der Erhebungsdauer von 61 Tagen betrug der Rücklauf 64\% entsprechend 2040 Fragebogen. Die Rücklaufrate in den 8 Bezirksvereinen schwankte zwischen 42 und 97\%. 85\% der retournierten Fragebogen wurden von Grundversorgern geliefert. 
Die Ärztegesellschaft des Kantons Bern ist in 8 Bezirksvereine und 52 Notfalldienstkreise gegliedert. Die Organisation des Notfalldienstes erfolgte bisher weitgehend autonom durch die Notfalldienstkreise, den kleinsten Organisationseinheiten. Die Einteilung der Notfalldienstkreise in bezug auf die Grösse der versorgten Bevölkerung, der Anzahl Ärzte* pro 1000 Einwohner und der geographischen Ausdehnung ist sehr heterogen (Abb. 1). Der kleinste Notfalldienstkreis dehnt sich über eine Fläche von etwa $4 \times 2 \mathrm{~km}$ aus (Muri-Gümligen in der Agglomeration Bern, im Bernischen Emmental erreicht der Notfalldienstkreis Langnau-Oberes Emmental etwa $30 \times$ $36 \mathrm{~km}$ und in den Berner Alpen der Notfalldienstkreis Meiringen-Haslital etwa $30 \times 36 \mathrm{~km}$. Im Durchschnitt stellen 16 Ärzte pro Notfalldienstkreis die notfalldienstliche Versorgung von knapp 20000 Einwohnern sicher. Die Streuung der Anzahl Notfalldienstärzte pro Notfalldienstkreis ist aber beträchtlich und variiert zwischen 3 und 52 Notfalldienstleistenden pro Dienstkreis unter Auslassung der hohen Zahl notfalldienstpflichtiger Ärzte der Stadt Bern.

77,5\% der notfalldienstleistenden Ärzte praktizieren in Notfalldienstkreisen mit weniger als 20 Notfalldienstärzten (Abb. 2).

\section{Demographie der notfalldienstleistenden Ärzteschaft}

Im gesamten Kanton Bern ist eine Dichte von 0,7 Notfalldienstärzten/1000 Einwohner, 1,0 Grundversorgern/1000 Einwohner und 1,8 Praktizierenden aller Fachrichtungen/1000 Einwohner festzustellen.

Überraschenderweise finden sich auch Notfalldienstkreise mit sehr tiefen Ärztedichten unmittelbar neben den städtischen Agglomerationen wie Bern, Langenthal und Burgdorf (Abb. 3).

Der Altersmittelwert der Notfalldienstleistenden beträgt kantonal 52 Jahre und ist somit vergleichbar mit anderen Kantonen und der ganzen Schweiz.

\section{Abbildung 1}

Einwohner im Notfalldienstkreis.

Mit der männlichen Schreibweise sind immer beide Geschlechter gemeint.

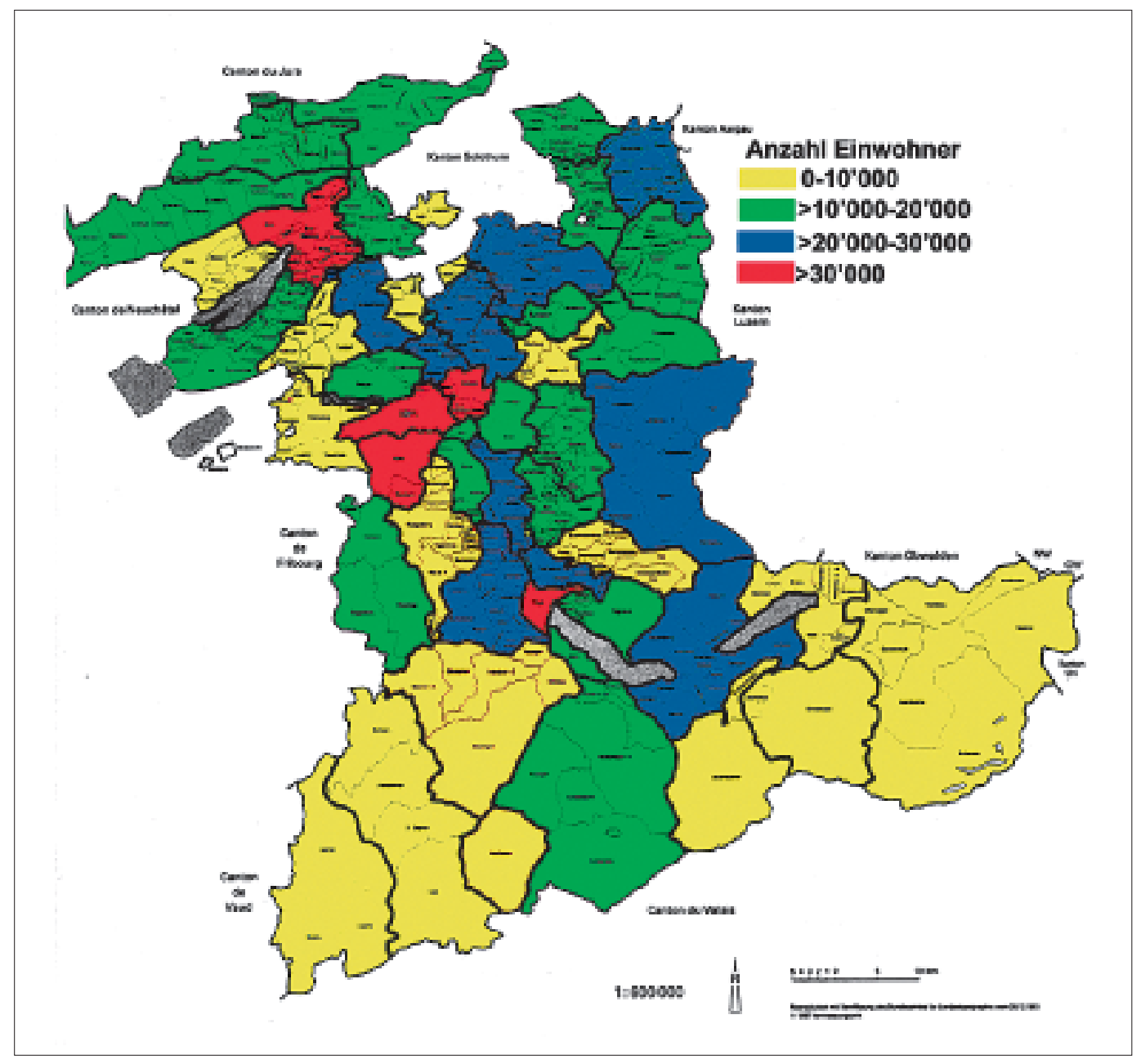


Abbildung 2

Anzahl eingeteilter Ärzte in den ND-Kreisen.

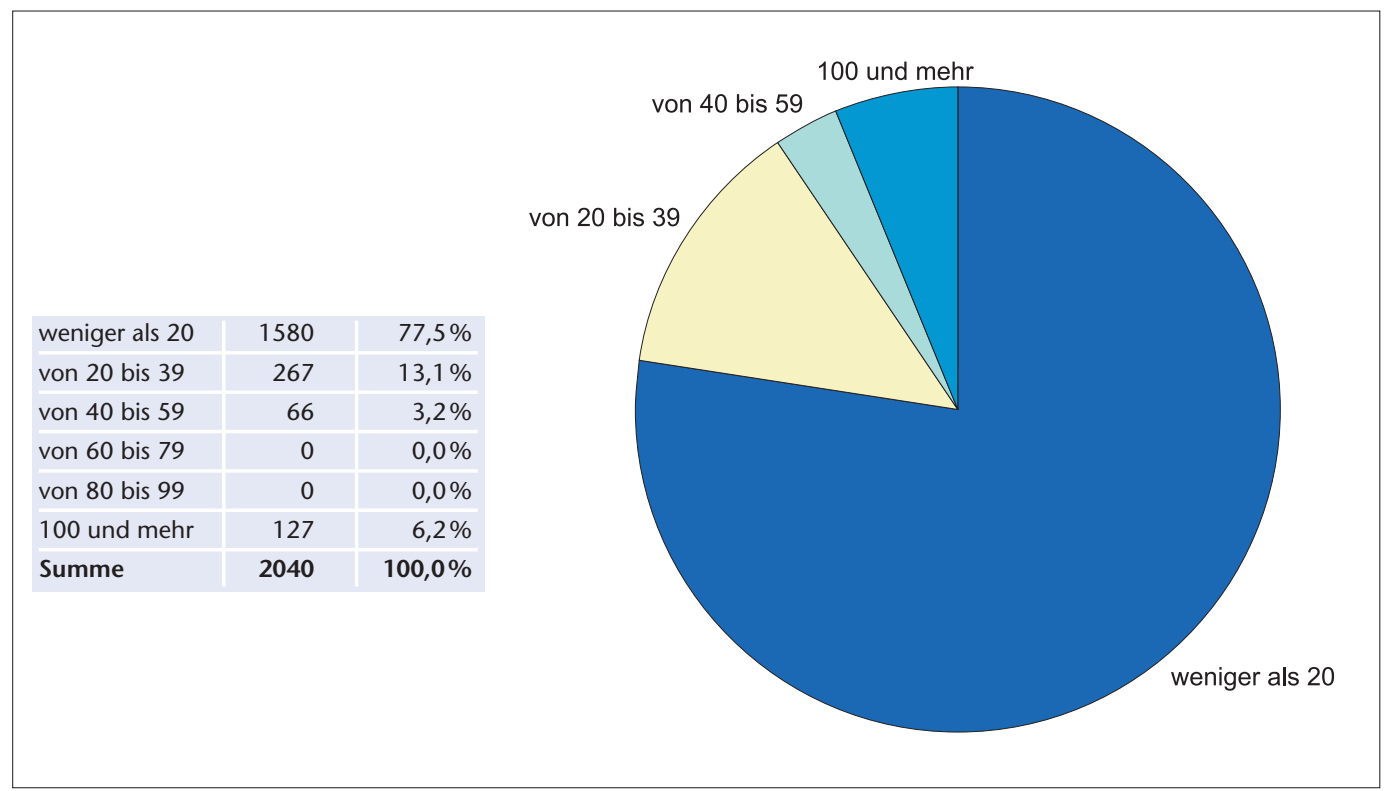

Abbildung 3

Anzahl Notfalldienstärzte pro 1000 Einwohner im Notfalldienstkreis.

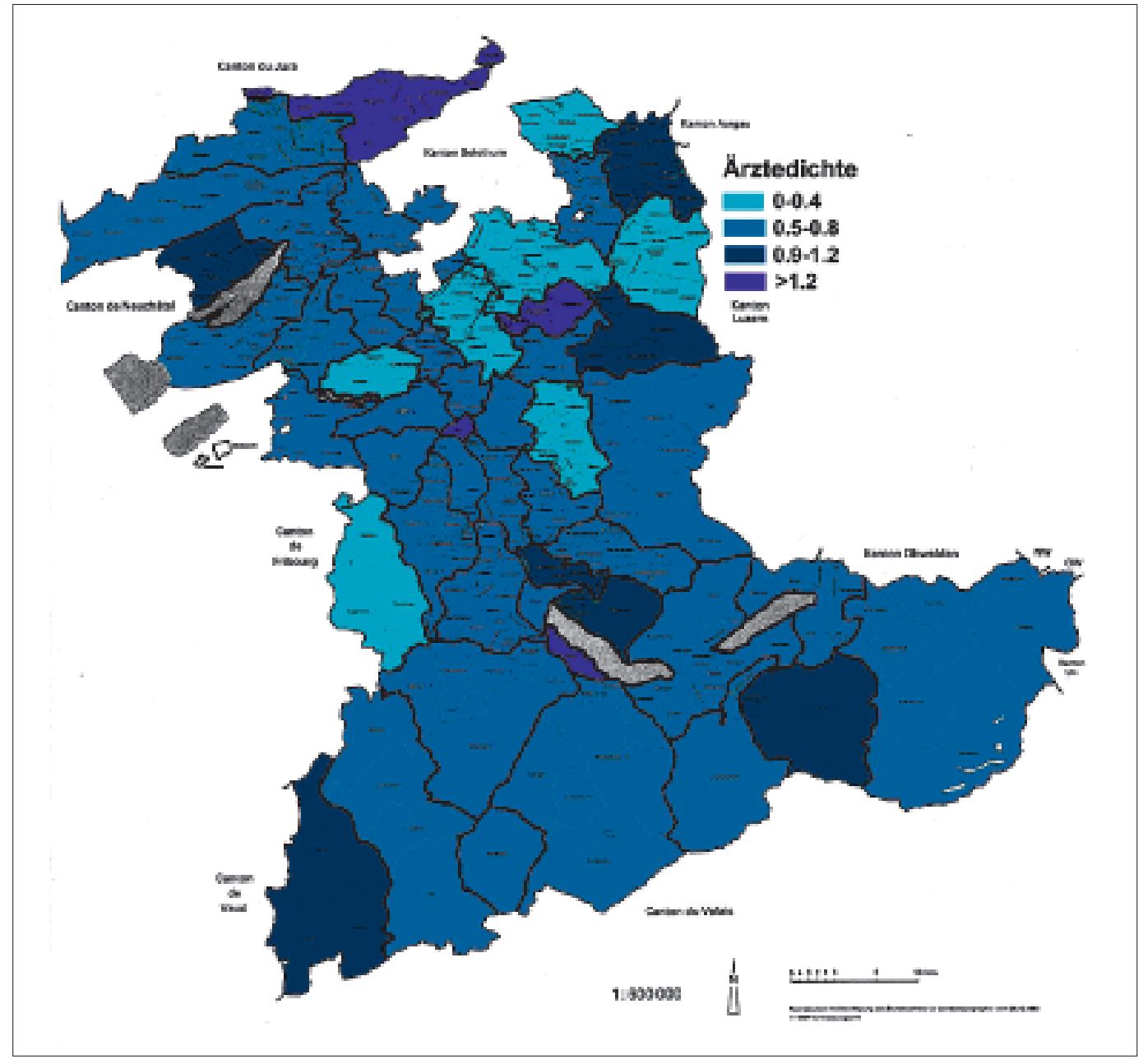


Altersverteilung der Teilnehmer pro Bezirksverein.

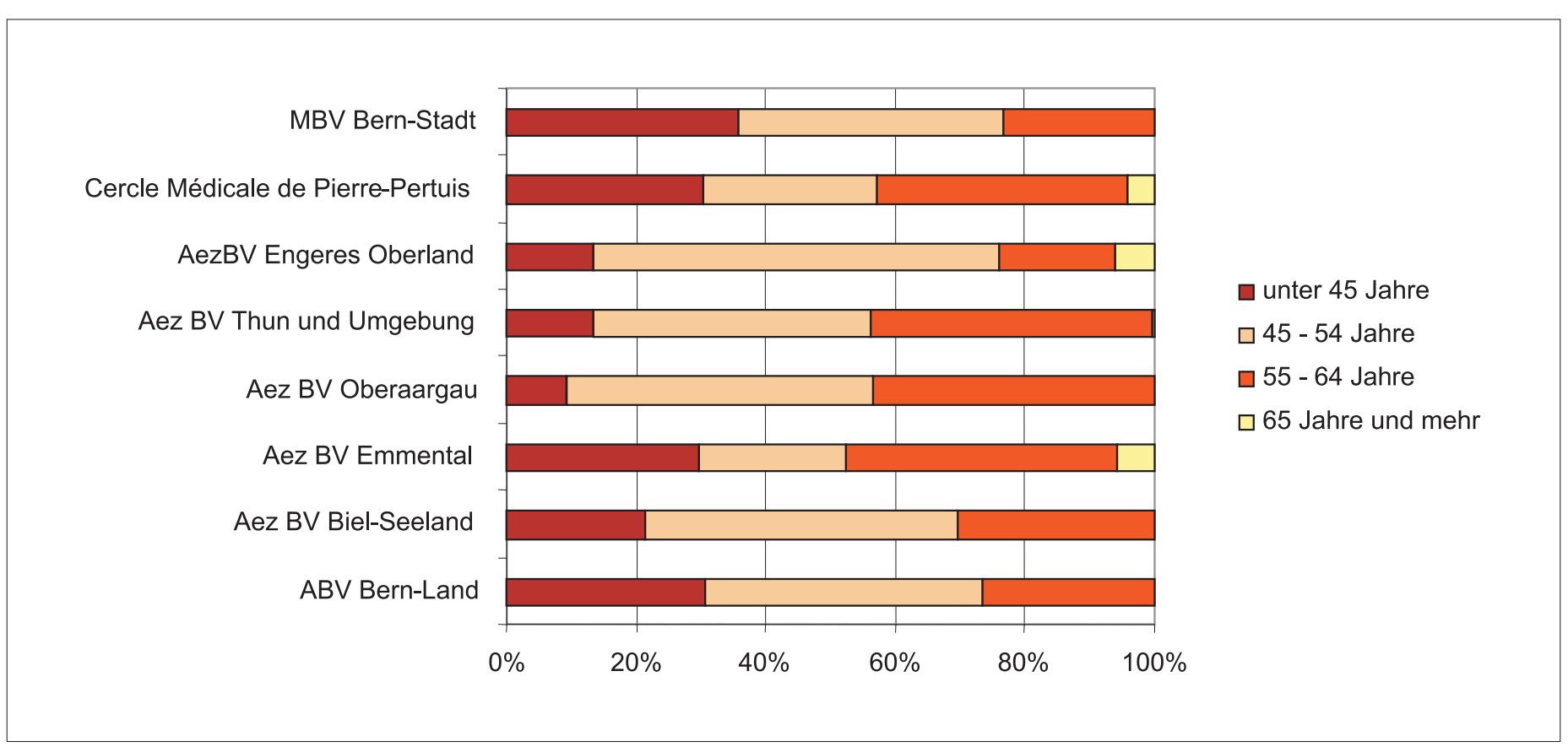

Es ist vorauszusehen, dass Bezirksvereine mit einem tiefen Anteil an Notfalldienstärzten unter 45 Jahren (z. B. in den Bezirksvereinen Thun und Umgebung, Engeres Oberland und Oberaargau) schon bald Probleme in der Organisation des ärztlichen Notfalldienstes haben werden (Abb. 4). Dies wird noch ausgeprägter bei einem zusätzlichen überdurchschnittlichen Anteil an über 65jährigen (z. B. in den Bezirksvereinen Emmental, Engeres Oberland und Pierre-Pertuis).

$11,4 \%$ der retournierten Fragebogen stammen von Notfalldienstärztinnen, 88,6\% von Notfalldienstärzten.

\section{Arbeitsbelastung im Notfalldienst}

Die Häufigkeit von Patientenkontakten im Notfalldienst ist eher niedrig (Abb. 5). So werden tagsüber in den Notfalldienstkreisen durchschnittlich nur 3,5 Patienten als Notfalldienst-

Abbildung 5

Durchschnittliche notfalldienstliche Patientenkontakte pro 24 Stunden und Notfalldienstkreis.

\begin{tabular}{|l|c|c|}
\multicolumn{2}{c}{ Mean } & \multicolumn{2}{c}{ Std. Dev. } \\
\hline Konsultation tags & 3.5 & 3.9 \\
\hline Konsultation nachts & 0.8 & 1.6 \\
\hline Hausbesuche tags & 0.4 & 0.9 \\
\hline Hausbesuche nachts & 0.3 & 0.6 \\
\hline Telefonanrufe tags & 2 & 3.3 \\
\hline Telefonanrufe nachts & 1 & 1.8 \\
\hline Patientenkontakte tags und nachts (Kons., Tel., Hausbesuche) & 8 & 7.6 \\
\hline
\end{tabular}

arzt behandelt und 2,0 notfalldienstliche Telefone erledigt. Auch die Rate der notfalldienstlichen Hausbesuche tagsüber ist mit 0,4 tief. Die Werte für nächtliche Patientenkontakte sind noch tiefer. Das Total für alle notfalldienstlichen Patientenkontakte pro 24 Stunden in den Notfalldienstkreisen beträgt 8,0. Dabei ist zu bemerken, dass die Streuung recht gross ist (Abb. 6). Kantonale Durchschnittswerte sind vorsichtig zu interpretieren, da sie nur eine beschränkte Aussagekraft über die individuelle Arbeitsbelastung der Notfalldienstärzte haben.

Die Anzahl der zu leistenden Notfalldiensttage pro Jahr variiert stark. In 21,7\% der Rückantworten werden 30 bis 39 Notfalldiensttage pro Jahr genannt. Ein Drittel der Antwortenden nennt 50 bis 300 und mehr Tage Notfalldienst pro Jahr. $\mathrm{Zu}$ beachten ist, dass in $7 \% 150$ bis 300 und mehr Notfalldiensttage genannt wurden (Abb. 7).

\section{Diskussion und Massnahmen}

Nach wie vor stellen im ganzen Kanton Bern die Grundversorger den ärztlichen Notfalldienst sicher. Sie sind in stark unterschiedlichem Masse durch die sich örtlich ergebende Präsenzzeit belastet. Zusammen mit der eher tiefen Rate an notfalldienstlichen Patientenkontakten (allerdings bei grosser Streuung) wird die Effizienz der notfalldienstlichen Versorgung fraglich. Noch unklar ist nämlich, wie oft Patienten eher andere notfalldienstliche Einrichtungen aufsuchen. 
Die Heterogenität in den Notfalldienstkreisen in bezug auf Anzahl beteiligter Ärzte, Grösse der betreuten Bevölkerung und Ausdehnung der Notfalldienstkreise ist sehr gross. Eine Vereinheitlichung muss in der Zukunft wohl angestrebt werden. Die ausgeprägt föderalen Organisationsformen mit Delegation der meisten organisatorischen Aufgaben auf das Niveau der Notfalldienstkreise ist aber den mehr grossräumigen, (über-)regionalen Änderungen nicht förderlich.

Stadt-Land-Unterschiede wurden in dieser Studie kaum gefunden. Keine Unterschiede konnten bezüglich Anzahl Einwohner pro NDKreis, Anzahl Notfalldienstärzte und Grundversorger/1000 Einwohner in den Bezirksvereinen bzw. Notfalldienstkreisen, notfalldienstlicher Patientenkontakte und der Altersstruktur gezeigt werden.

Geklärt werden muss, ob die Grundversorger in Zukunft noch bereit sind, den Notfalldienst in der bisherigen Form weiter zu leisten. Möglich- keiten, um den Notfalldienst mit den Grundversorgern weiterhin aufrechtzuerhalten, sehen wir z.B. in den folgenden Massnahmen:

- Zusammenschlüsse von Notfalldienstkreisen;

- sinnvolle Änderungen der Grenzziehung;

- Miteinbeziehen von gegebenen oder neu zu schaffenden Einrichtungen wie Spitalnotfallpforten mit oder ohne vorgelagerten Triageposten;

- modifizierte Hausarztposten auf Grundlage der holländischen Modelle;

- Erleichterung der Niederlassung in bestimmten Bezirksvereinen;

- Erhöhung der Attraktivität des Hausarztberufes;

- Förderung der Hausarztmedizin in der Ausbildung usw.

Wir Ärztinnen und Ärzte sind aufgefordert, mögliche Szenarien zu diskutieren und umzusetzen.

\section{Abbildung 6}

Anzahl Patientenkontakte pro 24 Stunden (Konsultationen, Hausbesuche, Telefone) in den Notfalldienstkreisen.




Abbildung 7

Anzahl zu leistender Notfalldiensttage pro Jahr.

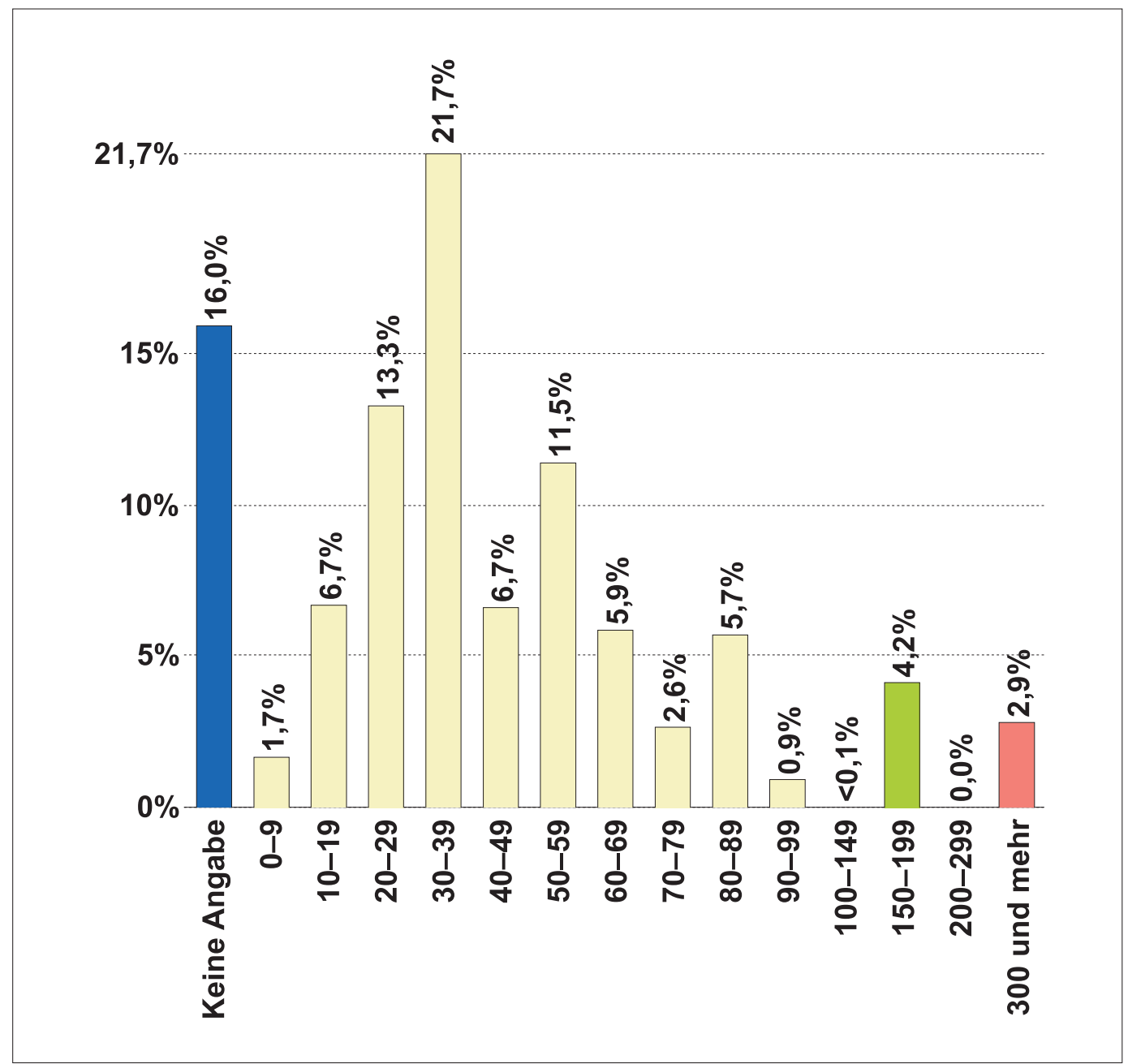

